\title{
The dust coma of the active Centaur P/2004 A1 (LONEOS): a CO-driven environment? ${ }^{\star}$
}

\author{
E. Mazzotta Epifani ${ }^{1}$, P. Palumbo ${ }^{2}$, M. T. Capria ${ }^{3}$, G. Cremonese ${ }^{4}$, M. Fulle ${ }^{5}$, and L. Colangeli ${ }^{1}$ \\ 1 INAF - Osservatoio Astronomico di Capodimonte, via Moiariello 16, 80131 Napoli, Italy \\ e-mail: epifani@na.astro.it \\ 2 Universitá Parthenope, via A. De Gasperi 5, 80133 Napoli, Italy \\ 3 INAF - Istituto di Astrofisica Spaziale, via del Fosso del Cavaliere 100, 00133 Roma, Italy \\ 4 INAF - Osservatorio Astronomico di Padova, Vicolo dell'Osservatorio 5, 35122 Padova, Italy \\ 5 INAF - Osservatorio Astronomico di Trieste, via Tiepolo 11, 34131 Trieste, Italy
}

Received 13 March 2006 / Accepted 2 August 2006

ABSTRACT

\begin{abstract}
Context. Centaurs reside in a region of the Solar System where many volatile species begin to have significant sublimation rates, and several examples of cometary activity have been observed in this group. The source of activity is not well known for most of the active Centaurs. If the sublimation rate of water is low, the sublimation of other volatiles, such as $\mathrm{CO}$, could drive the presence of a coma. Aims. The aim of this paper is to study the dust environment of the active Centaur P/2004 A1 (LONEOS), which was observed to have a significant coma and a very long perspective anti-tail (neck-line) at a heliocentric distance of 5.5 AU.

Methods. $R$-band images taken at the TNG telescope were used as input for an inverse numerical model, meant to derive the physical parameters of dust grains in the coma.

Results. The Centaur showed an asymmetric coma and a neck-line extending at least to $1.5 \times 10^{5} \mathrm{~km}$ in the solar direction, emitted half an orbit before the observation. The $A f \rho$ value measured in a $5^{\prime \prime}$ aperture is $334 \pm 15 \mathrm{~cm}$, indicating a significant dust production rate, comparable to that of several short-period comets at much lower heliocentric distances. The emitted grains are larger than $1 \mathrm{~cm}$, and the dust production rate has been around $100 \mathrm{~kg} \mathrm{~s}^{-1}$ during the last ten years. For $R_{n}=10 \mathrm{~km}$ and a maximun size of the uplifted grain $>3 \mathrm{~cm}$, a CO molecular abundance $Q>10^{30} \mathrm{~mol} \mathrm{~s}^{-1}$ is required if it is uniform over all the surface.
\end{abstract}

Key words. minor planets, asteroids - comets: individuals: P/2004 A1 (LONEOS)

\section{Introduction}

The Centaurs are a dynamical class of minor bodies in the Solar System, moving on highly chaotic orbits with perihelion lying between Jupiter's and Neptune's orbits. Their dynamical behaviour is still poorly understood: they are often considered to be objects originating in the Kuiper Belt (KB), especially from either the near Neptune high-eccentricity (NNHE) region, broadly similar to the so called "Scattered Disk" (Duncan \& Levison 1997), or else from the Plutinos population, as inferred from the possible Plutino origin of the Jupiter family comets (Yu \& Tremain 1999). Centaurs are in any case brief residents in the region between the gas giant planets, with calculated orbital lifetimes of less than $10^{7}$ years (Holman \& Wisdom 1993). Objects that survive the dynamical environment of this region may then become Jupiter family comets (Levison \& Duncan 1997).

As of January 2006, 80 objects have been classified as pertaining to the Centaur family. Their number growing is continual, thanks to the dedicated surveys of the ecliptic programmed

^ Based on observations collected at the Italian Telescopio Nazionale Galileo (TNG), operated on the island of La Palma by the Centro Galileo Galilei of the INAF (Istituto Nazionale di Astrofisica) at the Spanish Observatorio del Roque de los Muchachos of the Instituto de Astrofísica de Canarias, and at the Centro Astronómico Hispano Alemán (CAHA) at Calar Alto, operated jointly by the Max-Planck Institute für Astronomie and the Instituto de Astrofísica de Andalucia (CSIC). and executed with the aim of discovering and characterising new outer Solar System objects (Sheppard et al. 2000; Trujillo et al. 2001; Larsen et al. 2001; Millis et al. 2002; Petit \& Gladman 2003; Bernstein et al. 2004; Elliot et al. 2005; Petit et al. 2006). The physical properties of the Centaurs can provide information about the primordial processes that dominated the early solar nebula. Despite their usual intrinsic faintness, some Centaurs have been spectroscopically observed in the visible and the near-infrared (see e.g. Brown \& Koresko 1998; Brown 2000; Barucci et al. 2000, 2002; Lazzarin et al. 2003; Doressoundiram et al. 2003; Dotto et al. 2003a,b; Fornasier et al. 2004; Doressoundiram et al. 2005; Barucci et al. 2005). In some cases, near-infrared spectroscopic observations indicate the presence of a specific compound: water in (2060) Chiron (Brown \& Koresko 1998; Luu et al. 2000), in (5145) Pholus (Davies et al. 1993; Luu et al. 1994), in (1997 CU26) (10199) Chariklo (Brown \& Koresko 1998; Brown 2000; Dotto et al. 2003c) and very recently in (32532) Thereus (Merlin et al. 2005); methanol in (5145) Pholus (Cruikshank et al. 1998). Recently, radio wavelength measurements allowed only upper limits for the CO production in several Centaurs to be derived (Bockelée-Morvan et al. 2001). CO has been weakly detected in (2060) Chiron (Womack \& Stern 1999) and definitely observed in the coma of the giant comet 29P/Schwassmann-Wachmann 1 (29P/S-W 1) (Cochran et al. 1980; Senay \& Jewitt 1994; Festou et al. 2001), which is now dynamically classified as one member of the Centaur family. 
Table 1. Active Centaurs (as of January 2006).

\begin{tabular}{ccccccc}
\hline \hline & $q(\mathrm{AU})^{a}$ & $e^{b}$ & $i\left(^{o}\right)^{c}$ & $P(\text { year })^{d}$ & TJ $^{e}$ & $f$ \\
\hline 29P/S-W 1 & 5.724 & 0.044 & 9.4 & 14.7 & 2.984 & \\
39P/Oterma & 5.471 & 0.245 & 1.9 & 19.5 & 3.005 & \\
(2060) Chiron & 8.454 & 0.383 & 6.9 & 50.7 & 3.356 & 95P/Chiron \\
165P/LINEAR & 6.830 & 0.621 & 15.9 & 76.4 & 3.095 & C/2000 B4 \\
166P/NEAT & 8.564 & 0.384 & 15.4 & 51.9 & 3.285 & C/2001 T4 \\
167P/CINEOS & 11.788 & 0.269 & 19.1 & 64.8 & 3.527 & C/2004 PY42 \\
C/2001 M10 (NEAT) & 5.303 & 0.801 & 28.1 & 138 & 2.586 & \\
P/2004 A1 (LONEOS) & 5.463 & 0.308 & 10.6 & 22.2 & 2.963 & \\
(174P/)2000 EC 98 & 5.826 & 0.457 & 4.3 & 35.1 & 3.032 & $(60558)$ \\
P/2005 S2 (Skiff) & 6.398 & 0.197 & 3.1 & 22.5 & 3.076 & \\
P/2005 T3 (Read) & 6.202 & 0.174 & 6.3 & 20.6 & 3.045 & \\
\hline
\end{tabular}

${ }^{a}$ Perihelion distance; ${ }^{b}$ eccentricity; ${ }^{c}$ inclination; ${ }^{d}$ period $;{ }^{e}$ tisserand parameter; ${ }^{f}$ former or alternative designation; ${ }^{g}$ presently inactive; ${ }^{h}$ activity questioned (probable bad seeing).

Table 2. Orbital parameters of the active Centaur P/2004 A1 (LONEOS).

\begin{tabular}{ccccccc}
\hline \hline$a(\mathrm{AU})^{a}$ & $q(\mathrm{AU})^{b}$ & $e^{c}$ & $P(\text { year })^{d}$ & ${\text { Peri }\left({ }^{\circ}\right)^{e}}$ & Node $\left({ }^{\circ}\right)^{f}$ & $i\left(^{\circ}\right)^{g}$ \\
\hline 7.896 & 5.463 & 0.308 & 22.2 & 20.49 & 125.3 & 10.6 \\
\hline
\end{tabular}

${ }^{a}$ Semimajor axis; ${ }^{b}$ perihelion distance; ${ }^{c}$ orbital eccentricity; ${ }^{d}$ orbital period; ${ }^{e}$ the $\mathrm{J} 2000$ argument of perihelion; ${ }^{f}$ the $\mathrm{J} 2000$ longitude of the ascending node; ${ }^{g}$ inclination.

Actually, Centaurs reside in a region of the Solar System where many volatile species begin to have significant sublimation rates (Delsemme 1982; Crovisier 1999), and several examples of cometary activity have been observed among the group, as summarised in Table 1.

The presence of a resolved coma in (2060) Chiron was reported after its discovery (Meech \& Belton 1989; Hartmann et al. 1990) and fully analysed (Luu \& Jewitt 1990) in order to investigate the cause of its cometary activity, the implication in the coma structure of the great mass of the object compared to other comets, the physical process that controls the mass loss rate. Its dust environment has been characterised (Fulle 1994) by applying a numerical inverse model of comet dust tails that also takes the fast nucleus rotation into account. The activity of the giant comet 29P/S-W 1 has been fully analysed (Jewitt 1990). The coma is persistent, but its morphology changes on a short time scale (weeks). This poses interesting constraints in modelling the composition of the nucleus, especially in the view of the firm detection of highly volatile species in the coma (mainly $\mathrm{CO}$ ) that could drive its activity. Also in this case, the dust environment of this Centaur has been characterised by means of an inverse numerical model (Fulle 1992). The comet 39P/Oterma is currently inactive (Fernandez et al. 2001) and was seen to be active only before it was perturbed into a Centaur orbit in 1963 (Jupiter encounter: perihelion increased from 3.39 to 5.47 AU; Kronk 2005; Marsden 1962). Centaur C/2001 M10 (NEAT) (Lawrence et al. 2001) was reported perhaps active at its discovery, but there is some questions as to whether this was merely an effect of poor seeing. For the 165P/LINEAR (first designation C/2000 B4 LINEAR), observers also report some hints of cometary activity (Kusnirak \& Balam 2000). 166P/NEAT (first designation C/2001 T4 NEAT) activity has been firmly identified since its discovery as a comet (Pravdo et al. 2001). Its dust coma environment was deeply analysed by Bauer et al. (2003a). The coma colour gradient of this Centaurs is rather puzzling, since C/NEAT (2001 T4) is one of the reddest objects of this family observed to date (its $V-R$ colour in 2001 exceeded that of Pholus), so it does not comfortably fit into the known colour distribution. 167P/CINEOS (first designation C/2004 PY42) was firmly identified in a Centaur-like orbit and its activity observed at the large heliocentric distance of $12.4 \mathrm{AU}$ (Tegler et al. 2005). $\mathrm{P} / 2005$ S2 (Skiff) (Green 2005), discovered in the course of the LONEOS (Lowell Observatory Near-Earth Object Search) survey programme, and P/2005 T3 (Read) (Read \& Scotti 2005), discovered in course of the Spacewatch survey, are very faint active Centaurs, showing moderately condensed coma and shortly extending tail at discovery. Centaur (60558) $2000 \mathrm{EC}_{98}$ was inactive at least up to April 2003 (Rousselot et al. 2005), when photometry was not contaminated by any cometary activity down to magnitude $\simeq 27 / \operatorname{arcsec}^{2}$. A substantial coma was detected around this Centaur on December 30, 2005 (UAI, 2006) and monitored in subsequent days (Choi et al. 2006).

The object $\mathrm{P} / 2004$ A1 (LONEOS) was discovered on January 13, 2004 (Green 2004; Skiff et al. 2004; Descour et al. 2004) on CCD images taken in the course of the LONEOS programme with the $0.59-\mathrm{m}$ Schmidt telescope. The discovery was confirmed on 1.8-m telescope frames, where the object showed a $3^{\prime \prime}$ well-condensed coma and a $12^{\prime \prime}$ tail. The object was identified as a comet. Several subsequent observations (Ticha et al. 2004; Naves et al. 2004; Tesi et al. 2004; Buzzi et al. 2004; Cremaschini et al. 2004) allowed the orbital characteristics of the object to be defined as pertaining to the family of Centaurs. The orbital elements of $\mathrm{P} / 2004$ A1 (LONEOS) are reported in Table 2.

The question of activity among Centaurs is part of a wider debate on the activity of minor bodies at a great distance from the Sun. Several short-period comets have been observed with a coma and even a well developed dust tail at distances larger than 3.5 AU (the canonical distance of $\mathrm{H}_{2} \mathrm{O}$ sublimation) (Szabó et al. 2002; Meech et al. 2004; Licandro et al. 2000; Lowry \& Fitzsimmons 2001, 2005; Lowry \& Weissman 2003; Lowry et al. 1999, 2003). This long-distance activity has been observed also in long-period and new comets (e.g. Szabó et al. 2001), and most remarkable is the case of the comet C/1995 O1 (Hale-Bopp) with its activity at 13.1 AU from the Sun (Weiler et al. 2003) and at 18-20 AU (Cartwright et al. 1999). Even in the KB, cometary 
Table 3. Observing conditions for the active Centaur P/2004 A1 (LONEOS).

\begin{tabular}{cccccccc}
\hline \hline${\text { Observation date }(\mathrm{UT})^{a}}^{a}$ & Telescope & $r(\mathrm{AU})^{b}$ & $\Delta(\mathrm{AU})^{c}$ & $\alpha\left(^{\circ}\right)^{d}$ & ${\text { PA }\left({ }^{\circ}\right)^{e}}$ & ${\text { Seeing }(\operatorname{arcsec})^{f}}^{\text {Total exposure time }^{g}}$ \\
\hline 2005 Apr. 3 01:37 & TNG & 5.54 & 4.69 & 6.0 & 123.9 & 1.1 & 2400 \\
2005 May 15 20:50 & CAHA & 5.57 & 5.25 & 10.2 & 114.2 & 1.9 & 2400 \\
\hline
\end{tabular}

${ }^{a}$ UT time in the middle of the total exposure time (coadded image); ${ }^{b}$ heliocentric distance; ${ }^{c}$ geocentric distance; ${ }^{d}$ Sun-target-observer angle; ${ }^{e}$ position angle of the extended Sun-target radius vector; ${ }^{f}$ average of the mean FWHM of several sample stars from individual frames; ${ }^{g}$ resulting from coadding of the individual frames.

activity has been suggested as one of the possible explanations for the light curve variations of the TNO $1996 \mathrm{TO}_{66}$ at $45 \mathrm{AU}$ (Hainaut et al. 2000) and has been reported for 1999 TD $_{10}$ (Choi et al. 2002).

Observations presented in this paper have been collected in the framework of a large observing programme aimed at investigating the activity at distances far from the Sun and its possible origin on a large sample of Solar System minor bodies. In this paper we present the results of a complete analysis of the coma and the dust environment of the active Centaur P/2004 A1 (LONEOS). In Sect. 2 the observations and the reduction process are reported. Section 3 describes the results of the analysis of the coma morphology and characteristics. Section 4 describes the dynamical history of the Centaur, while the possible origins of Centaur activity are discussed in Sect. 5. Section 6 comments on Centaur's prominent dust features, while the results of the application of the inverse numerical model are reported in Sect. 7. Conclusions and perspectives for future work are given in Sect. 8.

\section{Observations and reduction}

P/2004 A1 (LONEOS) was observed on April 3, 2005 with the $3.52 \mathrm{~m}$ Telescopio Nazionale Galileo (TNG) at the Observatorio del Roque de los Muchachos (La Palma, Canary Islands) and on May 15, 2005 with the $2.2 \mathrm{~m}$ telescope at the Centro Astronómico Hispano Alemán (CAHA) at Calar Alto (Spain). TNG images were obtained with the DOLORES (Device Optimised for the LOw RESolution) instrument, equipped with a $2 \mathrm{k} \times 2 \mathrm{k}$ Loral thinned and back-illuminated CCD with $15 \mu \mathrm{m}$ pixels (pixel scale of $0.275 \mathrm{arcsec} / \mathrm{px}$ ) and the Cousins broadband filter $R$. CAHA images were obtained with the CAFOS (Calar Alto Faint Object Spectrograph) instrument, equipped with the $2 \mathrm{k} \times 2 \mathrm{k}$ SITE\#1d CCD with $24 \mu \mathrm{m}$ pixels (pixel scale of $0.53 \mathrm{arcsec} / \mathrm{px})$ and the Johnson broadband filter $R$.

In both cases, several long ( $>300 \mathrm{~s}$ ) exposures were obtained for the object, with the telescope tracking at the nonsidereal rate corresponding to the predicted motion of P/2004 A1 (LONEOS). All the images were bias-subtracted and flat-fielded in the standard manner, with the aid of the ESO's Munich Image Data Analysis System, MIDAS (1998). The bias value was obtained using bias frames with zero-second exposure. In both observing runs, a flat field was obtained from the average of several twilight sky exposures.

At TNG we obtained 2 long-exposure consecutive images of the Centaur: $1 \times 600 \mathrm{~s}, 1 \times 1800 \mathrm{~s}$. In both images the Centaur displayed a well-developed coma and a long, sharp taillike structure. Unfortunately, the non-sidereal tracking velocity in the $\delta$ direction in the shortest frame was half of the real one, but due to the slow motion of the object, this results in an image "distorted" only about 1.5 pixels in the $\delta$ direction. Therefore,

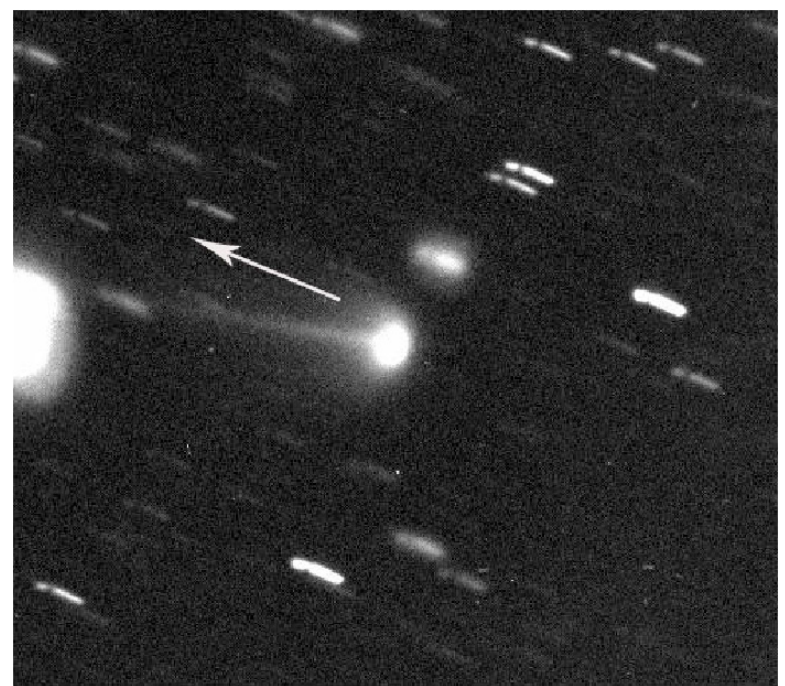

Fig. 1. TNG composite image of P/2004 A1 (LONEOS). Image width is $4.7 \times 10^{5} \mathrm{~km}$. N is up, E is right. The white arrow indicates the Sun direction. The grey scale has been adjusted to enhance coma structures and the presence of the neck-line.

we decided to sum both the 2 TNG images in order to increase the $\mathrm{S} / \mathrm{N}$ of the final image.

During the CalarAlto observing run, we obtained 4 longexposure consecutive images of the Centaur: $1 \times 300 \mathrm{~s}, 1 \times$ $600 \mathrm{~s}, 1 \times 1200 \mathrm{~s}, 1 \times 900 \mathrm{~s}$. A well-developed coma and the same sharp tail-like structure is present in the longest exposure. Unfortunately, some tracking problems during the last part of the observing sequence prevent also for this telescope the use of all the images in order to sum up and increase to its highest the SNR for the outer part of the coma. Only the first 3 frames were considered for composing of the final image.

The position of the Centaur optocentre in each single image selected for the coadding was determined by fitting a two-dimensional Gaussian to the innermost 50 pixels of the coma. The images were then recentred using this optocentre and summed. Sky correction was performed by subtracting a firstorder polynomial sky approximation computed from the pixel areas containing no stars. The observing conditions are summarised in Table 3. The final images are reported in Figs. 1 and 2.

To perform an absolute flux calibration of the comet images, appropriate fields from the list of Landolt (1992) (PG0918 and G1243 for the night at TNG; SA104 350 field, PG1530, and PG1633 for the night at CAHA) were observed at different airmasses during the observation nights. Both the observing nights were photometric, with the seeing value varying slightly from $1^{\prime \prime}$ to $1.2^{\prime \prime}$ throughout the night for the TNG observations and the seeing value stable around $1.9^{\prime \prime}$ for the CAHA observation. 


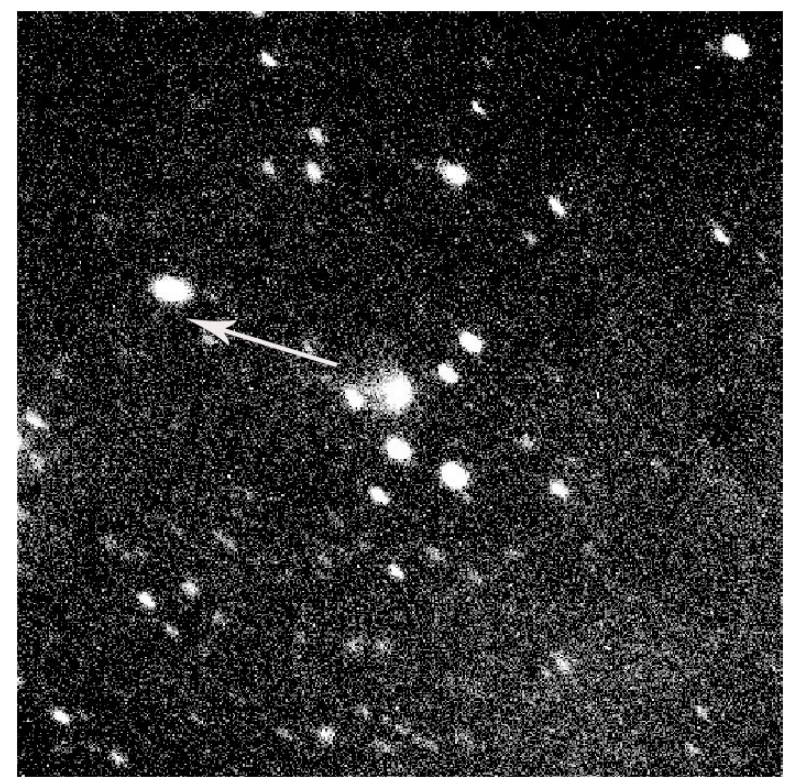

Fig. 2. CAHA composite image of P/2004 A1 (LONEOS). Image width is $1.9 \times 10^{6} \mathrm{~km}$. N is up, E is right. The white arrow indicates the Sun direction.

\section{Image analysis}

\subsection{TNG image}

The image obtained during the TNG run (Fig. 1) shows a very active object, with a well-developed coma and a long, sharp taillike structure elongated almost in the solar direction (perspective anti-tail). The coma is slightly asymmetric: it extends (counts above $1 \sigma$ of the background) up to $4 \times 10^{4} \mathrm{~km}$ in the $\mathrm{S}$ direction and up to $4.2 \times 10^{4} \mathrm{~km}$ in the $\mathrm{N}$ direction. Along the $\mathrm{E}-\mathrm{W}$ direction is even more asymmetric, due to action of the solar radiation pressure that distorts the shape of the external part of the coma: up to $2 \times 10^{4} \mathrm{~km}$ in the $\mathrm{E}$ direction (very close to the solar direction, see Table 3 ) and up $8.8 \times 10^{4} \mathrm{~km}$ in the $\mathrm{W}$ direction.

The thin spike clearly visible in the image extends at least up to $1.5 \times 10^{5} \mathrm{~km}$, but we can probably infer a longer extension (covered in the image by a trailed field star). It has a Position Angle (PA) of $281^{\circ}$, close to the sky-projected vector pointing to Sun, with $\mathrm{PA}=303.9^{\circ}$.

The $R$ magnitudes of the Centaur, derived in different optical apertures centred in the optocentre, are summarised in Table 4. The reduced magnitude $\left(H_{R}(1, \alpha)\right)$ is defined as:

$H_{R}(1, \alpha)=R-5 \log (r \Delta)$

where $R$ is the measured magnitude and $r$ and $\Delta$ (both in AU) are the heliocentric and geocentric distances at the time of observation. Available estimations of the effect of small-phase angles observations for few TNOs and Centaurs show that, down to phase angles of $0.1^{\circ}-0.2^{\circ}$, the magnitude follows a linear dependence vs. the phase (Belskaya et al. 2003). We calculated the absolute magnitude $H_{R}(1,0,0)$ with phase correction as:

$H_{R}(1,0,0)=R-5 \log (r \Delta)-\alpha \phi$.

In (2) $\alpha$ is the phase angle during the observations $\left({ }^{\circ}\right)$, and $\phi$ is the phase curve slope $\left(\mathrm{mag} /{ }^{\circ}\right)$. The $\phi$ values are not known for most of the Centaurs, since few objects have a well-sampled published phase curve (Bauer et al. 2002, 2003b). We decided to adopt the same value as Peixinho et al. (2004), $\phi=0.11 \pm 0.01$, obtained from a least-square fit of the linear approximation $\phi(\alpha)$ to the published data. The final uncertainity in the Centaur magnitude was derived from the photometric error $\sigma_{\text {calib }}$ in the calibration curve, taken as the rms of the standard stars fit.

We found published data on $R$ and $H_{R}$ for 5 of the 10 Centaurs reported or suspected to be active (excluding P/2004 A1 (LONEOS)), but only for 1 of them (C/NEAT 2001 T4; see Bauer et al. 2003a) do we have some indication of the aperture values used to derive the magnitudes, so a direct and precise comparison of absolute magnitude is impossible in the whole subset. As an indication, we observe that all the active Centaurs (except 39P/Oterma and C/2000 B4 LINEAR) have been reported with an absolute magnitude smaller than P/2004 A1 (LONEOS). The two darker Centaurs are, in any case, controversial (39P/Oterma is not presently active, and the activity of $\mathrm{C} / 2000 \mathrm{~B} 4$ LINEAR is in question). This means that among the firmly and presently active Centaurs, P/2004 A1 (LONEOS) is the darkest one in the $R$ band. Unfortunately, we do not have contemporary $V$ images of this Centaur, so we cannot infer anything about its visible colour. Activity in objects far from the Sun is usually associated with bluish colour; but this is not the case, for example, for C/NEAT (2001 T4), which is clearly active at 8.6 AU from the Sun and which presents one of the reddest colours among the minor bodies $(V-R=0.9$ in an aperture of $\left.10^{\prime \prime}\right) . \mathrm{P} / 2004 \mathrm{~A} 1$ (LONEOS) is also the active Centaur observed at the shortest distance from the Sun.

Our results for P/2004 A1 (LONEOS) show that, with respect to C/NEAT (2001 T4) (see Table 2 of Bauer et al. 2003a), the two Centaurs are similar in absolute brightness in all the considered aperture, even if C/NEAT is brighter.

A proxy for the dust production is the $A f \rho$ value (cm) (A'Hearn et al. 1984), where $A$ is the average grain albedo, $f$ the filling factor in the aperture field of view, and $\rho$ the linear radius of the aperture at the comet, i.e. the sky-plane radius. Af $\rho$, interpreted as the percentage of solar radiation scattered by the cometary dust towards the observer, can be obtained by means of the calculated photometric magnitude

$A f \rho=\frac{4 \cdot r^{2} \cdot \Delta^{2} \cdot 10^{0.4\left(m_{\mathrm{S}}-m_{\mathrm{C}}\right)}}{\rho}$

where $r$ is the heliocentric distance, $\Delta$ the geocentric distance, $m_{\mathrm{S}}$ the Sun magnitude in the $R$ band, and $m_{\mathrm{C}}$ the $R$ magnitude of the comet in the aperture of radius $\rho$.

Values of $A f \rho$ for P/2004 A1 (LONEOS), in different aperture radii, are shown in Table 4. A monotonical decrease in Af $\rho$ values with the nucleocentric distance $\rho$ may indicate nonsteady-state dust emission and possibly dust grain fading or destruction, as reported for the Centaur 166P/NEAT (Bauer et al. 2003a) and for several comets, such as C/2000 WM1 (LINEAR) (Lara et al. 2004). Conversely, for an ideal steady-state coma, Af $\rho$ should be constant at all apertures. Values for P/2004 A1 (LONEOS), reported in Table 4, are slightly different at different cometocentric distances, but a clear monotonical trend cannot be identified and most values are compatible within measurements errors. Therefore we can conclude that the dust environment of P/2004 A1 (LONEOS), as derived from analysis of coma photometry, is consistent with a scenario of steady-state emission.

Our results for this Centaur indicate a significant dust production rate: values found at a heliocentric distance of $5.5 \mathrm{AU}$ are of the same order of those found for several active comets at much lower heliocentric distances: 19P/Borrelly, 400-500 cm at 1.4 AU (Schleicher et al. 2003); 81P/Wild 2, 350 cm at $2.1 \mathrm{AU}$ (Schulz et al. 2003); 21P/Giacobini-Zinner, 300-400 cm at $1 \mathrm{AU}$ (Lara et al. 2003); 67P/Churyumov-Gerasimenko, $\sim 400 \mathrm{~cm}$ at 
Table 4. Magnitude of the active Centaur P/2004 A1 (LONEOS).

\begin{tabular}{cccccccc}
\hline \hline Observing night & Telescope & $d\left(^{\prime \prime}\right)^{a}$ & $R$ & $H_{R}(1, \alpha)$ & $H_{R}(1,0,0)$ & $\log _{10} \rho(\mathrm{km})^{b}$ & $A f \rho(\mathrm{cm})$ \\
\hline 2005 Apr. 2 & TNG & 3 & $19.19 \pm 0.05$ & $12.12 \pm 0.05$ & $11.46 \pm 0.05$ & 3.67 & $309 \pm 14$ \\
& & 5 & $18.56 \pm 0.05$ & $11.48 \pm 0.05$ & $10.82 \pm 0.05$ & 3.92 & $334 \pm 15$ \\
& & 7 & $18.22 \pm 0.05$ & $11.15 \pm 0.05$ & $10.49 \pm 0.05$ & 4.08 & $324 \pm 15$ \\
2005 May 15 & CAHA & 10 & $17.97 \pm 0.05$ & $10.89 \pm 0.05$ & $10.23 \pm 0.05$ & 4.22 & $288 \pm 13$ \\
& & 5 & $19.64 \pm 0.06$ & $12.31 \pm 0.06$ & $11.19 \pm 0.06$ & 3.76 & $232 \pm 13$ \\
& & 7 & $18.95 \pm 0.06$ & $11.62 \pm 0.06$ & $10.50 \pm 0.06$ & 3.98 & $263 \pm 15$ \\
& & 10 & $18.22 \pm 0.06$ & $10.89 \pm 0.06$ & $9.77 \pm 0.06$ & 4.28 & $257 \pm 14$ \\
\hline
\end{tabular}

${ }^{a}$ Aperture diameter; ${ }^{b}$ linear radius of the aperture at the comet.

1.2 AU (Weiler et al. 2004). P/2004 A1 (LONEOS) is not the more active Centaur observed: $\mathrm{C} / 2001 \mathrm{~T} 4$ (NEAT) in fact showed similar $A f \rho$ values at the greater heliocentric distance of 8.6 AU (Bauer et al. 2003a).

\subsection{CAHA image}

Even with lower general quality, the image obtained during the CAHA run (Fig. 2) also shows an active object, with a developed coma and a thin, sharp tail-like structure, elongated almost in the solar direction (prospectic anti-tail). The morphological analysis of this image shows that in this case the coma also appears slightly asymmetric, even if the geometry of the observation is slightly changed. The coma is more asymmetric in the N-S direction, extending (counts above $1 \sigma$ of the background) up to $3 \times 10^{4} \mathrm{~km}$ in the $\mathrm{N}$ direction and up to $4.4 \times 10^{4} \mathrm{~km}$ in the $\mathrm{S}$ direction. Along the $\mathrm{E}-\mathrm{W}$, it appears more compact with respect to the TNG image, and also more symmetric, extending up to $3.6 \times$ $10^{4} \mathrm{~km}$ in the $\mathrm{E}$ direction (very close to the solar direction, see Table 3) and up $3.2 \times 10^{4} \mathrm{~km}$ in the $\mathrm{W}$ direction.

The thin spike is less clearly visible with respect to the TNG image, but it can be considered persistent as its extension can be measured at least up to $1.5 \times 10^{5} \mathrm{~km}$. The sky-projected vector pointing to the Sun has $\mathrm{PA}=279^{\circ}$.

The magnitude values in different apertures are reported in Table 4. The Centaur appears darker with respect to the TNG observing run, as expected from the (slight) increase in the heliocentric distance (see Table 3). The Af $\rho$ values for P/2004 A1 (LONEOS) observed from CAHA, as can also be seen in Table 4, are still consistent with a steady-state dust coma, even if there is a general decrease in the absolute value due to the increase of the heliocentric distance and to a consequent expected decrease in the dust activity. They are still compatible with values found for several active comets at much shorter heliocentric distances: e.g., 81P/Wild 2, $200 \mathrm{~cm}$ at $2.6 \mathrm{AU}$ (Schulz et al. 2003) and $\sim 230 \mathrm{~cm}$ at $1.6 \mathrm{AU}$ (Farnham \& Schleicher 2005); $\mathrm{C} / 2000 \mathrm{WM} 1, \sim 200 \mathrm{~cm}$ at 1.21 AU (Lara et al. 2004).

Due to the lower SNR value of the CAHA image, the following analysis (see Sects. 6 and 7) was only performed on the TNG image.

\section{Dynamical history of the Centaur}

The dynamical history of P/2004 A1 (LONEOS) was reconstructed by means of the Horizons Ephemeris System. Figure 3 shows the trend for some of the Centaur orbital parameters from 1900 to the present day. It is possible to see that the orbit of P/2004 A1 (LONEOS) has been quite stable up to July 1992, when a close encounter with Saturn (July 30th; distance $d=$ $0.03 \mathrm{AU}$ ) caused a sudden change in the orbital parameters.
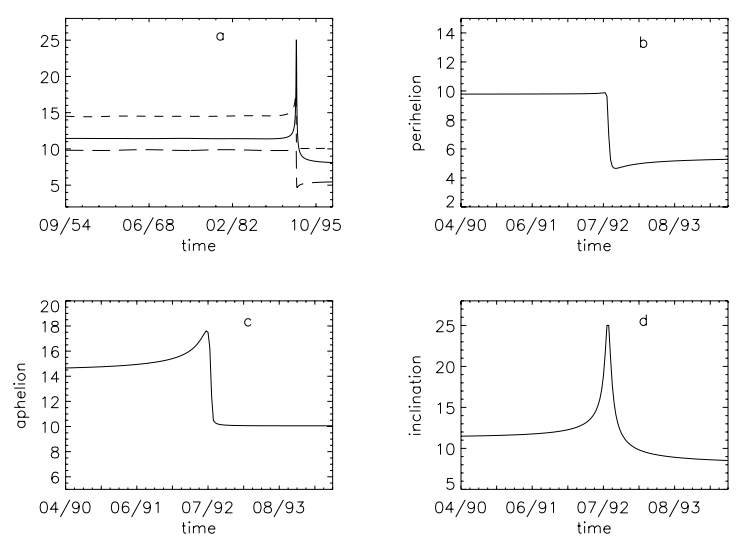

Fig. 3. Trend in the orbital parameters of $\mathrm{P} / 2004$ A1 (LONEOS). a). Trend in perihelion distance (long-dashed line), aphelion distance (dashed line), and inclination (continuous line) from 1954 to the observation time. b). Expanded version of top left graphics from 1990, for perihelion distance. c). Same as b) for aphelion distance. d), same as b) for inclination.

Table 5 summarises the main orbital changes: the perihelion distance dropped to 4.8 AU and then slowly increased to 5.5 AU (TNG-CAHA observing period). The semimajor axis dropped to 7.9 AU (TNG-CAHA observing period), while the orbit changed its shape and orientation. The observations presented in this paper therefore refers to the first passage of this object at its reduced perihelion distance.

To investigate the possible activity of the Centaur before the Saturn encounter and the subsequent decrease in the perihelion distance, we searched for the object in the Palomar Digitized Sky Survey plates back to 1950. Only three "candidate" plates were found (i.e., plates including the sky region where and when the Centaur was expected to be); and, among these, only plate ER732 (UK Schmidt), taken on July 11, 1988 when the Centaur was at 10.8 AU from the Sun, shows a not clearly identified object (with respect to the USNO stellar map). The sky region was analysed using other stellar catalogues (Tycho-2, 2MASS) to confirm or exlude the DSS identification, but unfortunately no clear identification of the presence of P/2004 A1 (LONEOS) was possible. Therefore we cannot speculate on the influence of the Saturn encounter and of the resulting sudden change in the orbital parameter on the dust activity of the Centaur.

\section{Sources of distant activity}

The source of activity is not known for most of the active Centaurs. The fundamental causes believed to induce global or 
Table 5. Changes in the P/2004 A1 (LONEOS) orbital parameters due to Saturn encounter.

\begin{tabular}{cccc}
\hline \hline Parameter & Before encounter & After encounter & Observing period \\
\hline Perihelion distance (AU) & 9.78 & 4.79 & 5.46 \\
Semi-major axis (AU) & 12.17 & 7.51 & 7.92 \\
Aphelion distance (AU) & 14.56 & 10.22 & 10.37 \\
Eccentricity & 0.20 & 0.36 & 0.31 \\
Inclination $\left(^{\circ}\right)$ & 11.39 & 17.73 & 8.02 \\
\hline
\end{tabular}

local changes on the surface of these bodies are thermal evolution by insolation and collisions among the same objects.

The activity at distances from the Sun greater than 3-4 AU cannot be explained with water sublimation (Meech \& Svoren 2005), but could be better explained with the sublimation or the release of very volatile gases, in particular CO. Probably abundant in the cold outer regions of the presolar nebula in which comets formed, $\mathrm{CO}$ is relatively abundant in comet nuclei (till 24\% relative to water in some comets) (Bockelée-Morvan et al. 2005). The CO and other volatiles can be present in the nuclei not only as pure ices, but also as gases trapped in the cells of amorphous water ice (Capria et al. 2000a,b). In the first case, a gas flux that could be able to sustain various degrees of activity could be found even at a very large heliocentric distance, as the sublimation of $\mathrm{CO}$ ice begins at $25 \mathrm{~K}$. If the $\mathrm{CO}$ is trapped as a gas in the amorphous water ice, it would be released when the irreversible transition from amorphous to cristalline state takes place, at temperatures over $100 \mathrm{~K}$ (Prialnik et al. 1995; Jenniskens et al. 1998). Also in this case, the trapped gas could be relatively abundant: water ice has the ability to trap gases up to a gas-to-ice ratio of 3.3 , as seen in laboratory experiments (Laufer et al. 1987).

Cometary activity can be studied from a theoretical point of view through a mathematical model of the thermal evolution and differentiation of the nucleus (Coradini et al. 1997; Capria 2002). This kind of model solves the heat transport and the gas diffuse equations in an idealised nucleus, depicted as a porous sphere composed of a mixture of dust and different ices. From the results of this model, applied several times to the simulation of real comets, Centaurs and Kuiper Belt Objects (Capria et al. 2000a,b, 2002), we can see that, both in the case of CO present as an ice and in the case of $\mathrm{CO}$ present as a trapped gas, the source of a CO-driven activity should be found under the surface, where the internal temperatures could drop quickly to very low values. Another characteristic of CO-driven activity that can be derived from the theoretical models is that $\mathrm{CO}$, when it is present, tends to flow from the body along most of its orbit and from everywhere on the surface, due to the low sublimation temperature and the depth of sublimation and transition fronts. The sublimation front, in particular, would be at a quasi-constant temperature.

The dust release from the nucleus surface due to $\mathrm{CO}$ drag is then expected to be different from that due to water: $\mathrm{CO}$, and then the associated dust flux, is expected to leave the comet nucleus uniformly all over the nucleus surface, on both the night and day sides. Also, since it is coming from the nucleus interior, it is expected that its loss rate can change very slowly in time after its onset of activity.

\section{The P/2004 A1 (LONEOS) antitail}

Figure 4 shows isophotes of the TNG image of P/2004 A1 (LONEOS) (Panels a to $\mathbf{c}$ refer to different $(u, w)$ combinations see Sect. 7 for details). The narrow tail clearly visible in the

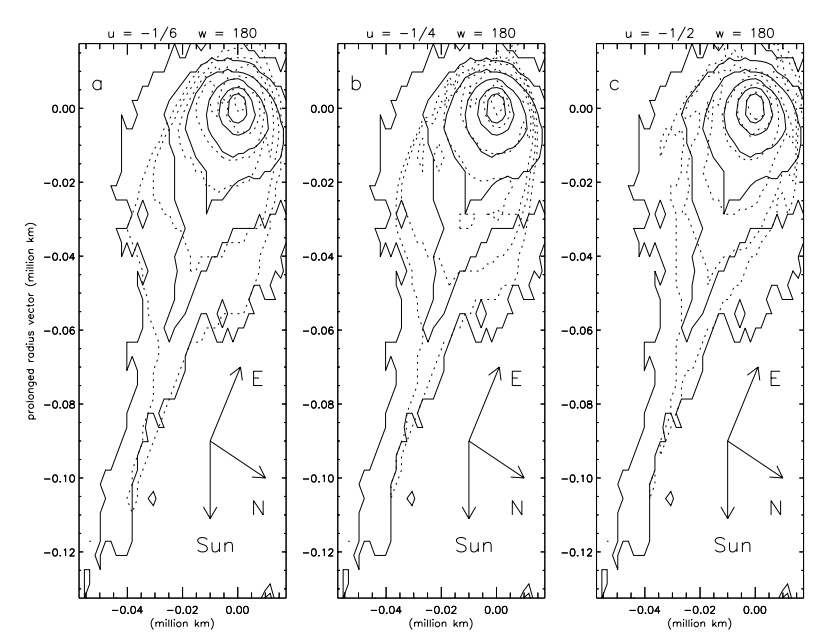

Fig. 4. Observed (continuous lines) and calculated (dashed lines) isophotes of $\mathrm{P} / 2004 \mathrm{~A} 1$ (LONEOS) in the TNG image. Levels of the isophotes are 21.33, 22.08, 22.83, 23.58, 24.34, and $25.09 R$-mag $\operatorname{arcsec}^{-2}$. The Sun-pointing sky-projected vector, the N and $\mathrm{E}$ directions are shown. Panels a) to c) refer to different $(u, w)$ combinations (see text for more details).

image is a perspective antitail composed of dust. In Fig. 4, the orbit already covered by $\mathrm{P} / 2004 \mathrm{~A} 1$ (LONEOS) has a PA $=284.5^{\circ}$, i.e. is a vector pointing towards bottom-left making an anticlockwise angle of $160.5^{\circ}$ with respect to the y-axis. It does not fit the narrow tail: the comet orbit lies to the right of the tail with a PA difference of $3^{\circ}$. A possible trail, due to the constant dust release of boulders during many orbits, does not necessarily lie along actual Centaur orbit, due to strong orbital perturbations by Saturn during the previous aphelion passage.

A classical dust tail is possible in the angular sector (on the left in Fig. 4) between the antisolar direction $(+y$ direction in Fig. 4) and the orbit covered by the comet, so that the observed narrow tail lies within this sector. In dust antitails, the closer the tail to the comet orbit, the larger the grains composing it, and the older the dust ejection that has generated the tail. In this particular case, the synchrone-syndyne model provides an ejection time close to the previous aphelion, which occurred about ten years before the observation. It is evident that only very large grains can remain close to the comet nucleus (at distances less than $1.5 \times 10^{5} \mathrm{~km}$ ) for such a long time. The synchrone-syndyne model provides $\beta$ parameters lower than $10^{-4}$, i.e. dust sizes surely larger than $1 \mathrm{~cm}$, and up to $10 \mathrm{~cm}$ for the tail section merging to the coma (see next section for details on $\beta-d$ conversion).

The synchrone-syndyne model suggests only approximate results, because it does not take into account that dust leaves the coma with a non-zero ejection velocity. A realistic dust coma model taking such a parameter into account allows us to interpret the narrow tail in terms of a neck-line, which is consistent with a steady dust release along most of the comet orbit. In fact, 
neck-lines are observed as narrow and straight tails with a precise orientation. In the P/2004 A1 (LONEOS) case, the computed PA of the neck-line fits the PA of the observed narrow tail within $1^{\circ}$.

Kimura \& Liu (1977) were the first to introduce the idea of neck-lines to explain the most prominent observable dust structures in cometary tails when the Earth is close to the orbital plane. In the following, we give a short description of processes forming a neck-line. For a more detailed description, the reader is referred to Fulle et al. (2004). A so-called synchronic "tube" is a theoretical dust tail composed of any possible $\beta$ parameter (i.e. dust size) and ejected at a given time. The real dust tail is simply the superposition of all synchronic tubes ejected at all times before the tail observation. The synchronic tube section is related to the dust ejection velocity: for times very close to the observation, such a section is a circle with the radius given by the product of the dust ejection velocity times the time interval between dust ejection and observation. As we go far back in time from the observation, the section of the synchronic tubes are no longer circles: sun tides become important, changing the shape of the tube section.

In order to compute the shape of the tube section, it is necessary to consider the motion of each dust grain composing the tube. Let us start with grains with $\beta=0$. They move on orbits very close to the comet orbit: the only difference is due to the ejection velocity vector of each grain, which (when it does not exactly lie in the comet orbital plane) will put the grains on orbits lying on planes inclined with respect to the comet orbital plane. Therefore, all grain orbits lie on planes that cross the comet orbital plane along the same nodal line, which must necessarily cross the Sun and the point (which is the same for all grains) where dust was ejected. All grains must cross the nodal line again, and the comet orbital plane too, at the point of the orbit opposite the ejection with respect to the Sun. There, all grains cross the comet orbital plane at almost the same time, shrinking into a 2D structure, which is centred on the comet nucleus when $\beta=0$. When $\beta>0$, its centre is shifted farer from the Sun, building up a structure that is no longer a 3D tube with a 2D section, but a 2D structure flat on the comet orbital plane. When the Earth is close to the comet's orbital plane, such a shrunk tube becomes brighter than the other 3D synchronic tubes, because the same amount of dust is concentrated in an infinitesimal sky area. Such a 2D synchronic tube, flat in the comet orbital plane, appears as a thin and straight tail, the neck-line.

Every observation has its own neck-line: the dust ejected at the point of the comet orbit exactly opposite the Sun is crossing the comet orbital plane building up the neck-line. When the observation occurs with the Earth far from the comet orbital plane, the neck-line has the same surface brightness as the surrounding tail, so it is not detected. In contrast, when the Earth is close to the comet's orbital plane, the neck-line is much brighter than the surrounding tail, because the same dust is concentrated in an infinitesimal sky area. In the P/2001 A1 (LONEOS) case, the angle between the comet orbital plane and the Earth-Comet vector was only $2^{\circ}$, which is a perfect condition for a neck-line appearance. It is therefore probable that the observed narrow tail is due to continuous dust ejection and that sky noise made it impossible to detect the dust tail covering the whole wide-angular sector between the narrow tail and the $+y$ direction in Fig. 4 . A 3D numerical model discussed in the next section confirms all these conclusions: a neck-line made of grains larger than $1 \mathrm{~cm}$ and released at a loss rate constant in time fits the observed tail perfectly.

\section{Application of the numerical dust tail model}

In this section we check whether the neck-line model is the most probable one for fitting the observed tail. This cannot be done by a direct dust tail model, i.e. changing all possible input parameters and checking which is the best fit, because the high number of parameters in every realistic dust model implies that hundreds of parameter combinations, not necessarily similar among them, would provide fits of very similar quality. Therefore, we adopt a regularized inverse approach that ensures we reach the most probable fit (with a least-square criterium). Such an approach characterises the inverse dust tail model, extensively described in Fulle (1989) and Epifani et al. (2001). The approach consists of two main steps: (i) computing the model dust tail and (ii) fitting it to the observed tail and deriving the dust parameters. The solution of the fit gives sampled values of the dust loss rate, $\dot{M}(t)$, of the size distribution $n(d, t)$, and of the $A f \rho$. Other non-linear parameters must be determined by a trial-and-error procedure:

(i) the dust ejection velocity, $v\left(t, d_{0}\right)$, that describes the time evolution of the dust ejection. Here $t$ is the time of dust ejection from the inner coma and $d_{0}$ a reference diameter;

(ii) the power index, $u=\partial \log v(t, d) / \partial \log d$, that characterises the power-law dependence of the dust velocity on the grain diameter $d: v(t, d)=v\left(t, d_{0}\right) \cdot\left(d / d_{0}\right)^{u}$;

(iii) the dust ejection anisotropy parameter, $w$, that is the half width of the Sun-pointing dust ejection cone.

Such an approach allows us to greatly reduce the number of tests necessary to ensure the uniqueness of the output parameter combination, because all the linear parameter are automatic outputs of the code, and only the combinations of the non-linear parameters must be investigated. Since the time-dependent dust loss rate and the time and size-dependent dust distribution are linear parameters of the model, the uniqueness of the output is stabler than in every direct approach. In the inverse approach, only the time- and size-dependent dust ejection velocity vector must be found by means of a trial and error procedure. Therefore, this quantity must be constrained by physical considerations, independent of the dust tail model, which we can derive taking into account considerations discussed in Sect. 5.

As a probable driver of the distant cometary origin, $\mathrm{CO}$ and associated dust flux is expected to leave the nucleus surface both on the night and day sides. Therefore, we used a value of $180^{\circ}$ for the $w$ parameter, which means isotrope ejection. We consider those that remain almost constant in time as the most probable solutions of a dust tail model. The dust ejection velocity is assumed to be constant in time. Its size-dependence is tested by the $u$ parameter: $u=-1 / 2$ is expected for perfectly spherical grains, $u>-1 / 2$ is expected for more and more aspherical grains, and $u=-1 / 6$ is the upper limit ever obtained by means of photometric analysis of neck-lines. Many inverse tail model applications in the past have shown that dust tail brightness data are unable to uniquely constrain the $u$ parameter. Changes in the outputs related to different $u$ input values allow us to estimate thfe uncertainty affecting the model solutions. In Fig. 5 we show the parameters of the inverse dust tail model fitting the brightness data shown in Fig. 4 (dashed isophotes). For $u=-1 / 6$ the dust velocity is almost independent of the dust size, as it is expected for very aspheric grains and/or in case dust fragmentation is occurring during the gas drag (Fulle et al. 1993). The model dust tail is built-up, ejecting tens of million of test dust particles along the orbit starting from the past aphelion. The true comet anomaly was $18.3^{\circ}$ at the observation, so that the neck-line was ejected at a true anomaly $18.3^{\circ}$ after the aphelion. The output parameters 


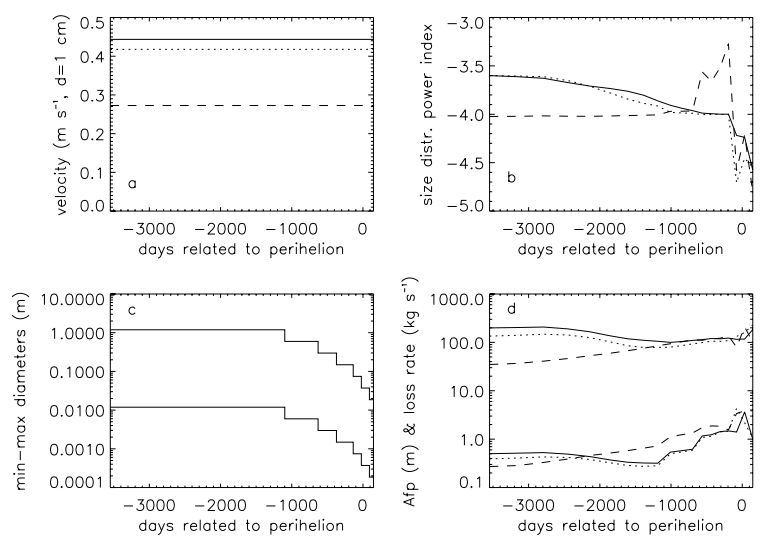

Fig. 5. Dust environment of $\mathrm{P} / 2004$ A1 (LONEOS) derived from the modelling of the TNG image. Dust ejection velocity a), power index of the differential size distribution b), size interval to which all the shown outputs are related c), mass loss rate and $A f \rho$ values $\mathbf{d})$. The line styles refer to different sets of parameters: $u=-1 / 6$ and $w=180^{\circ}$ (continuous line); $u=-1 / 4$ and $w=180^{\circ}$ (dotted line); $u=-1 / 2$ and $w=180^{\circ}$ (short dashed line) (see text for details on $u$ and $w$ values).

were sampled at time steps of about 100 days, so that we cannot investigate possible changes in the dust environment on shorter time scales.

Panel $\mathbf{c}$ of Fig. 5 shows which time-dependent $\beta$ parameter range allows us to fit the observed dust tail. Since we are always dealing with cm-sized grains, we have already converted the $\beta$ parameter to sizes, by means of Eq. (4) (for more details, see for example Krishna Swamy 1986):

$\beta=\frac{C_{\mathrm{pr}} Q_{\mathrm{pr}}}{\rho_{\mathrm{d}} d}$

where $C_{\mathrm{pr}}$ (depending on light velocity, solar mass, and $G$ ) is equal to $1.19 \times 10^{-3} \mathrm{~kg} \mathrm{~m}^{-2}$ and $Q_{\mathrm{pr}}$ is the dust scattering efficiency of grains of diameter $d$ and bulk density $\rho_{\mathrm{d}}$. In such a conversion, a dust bulk density of $10^{3} \mathrm{~kg} \mathrm{~m}^{-3}$ was adopted, and an increased value of the dust bulk density will determine a corresponding decrease in dust size. Also, a dust scattering efficiency $Q_{\mathrm{pr}}=1$ was adopted, as appropriate for the tail of $\mathrm{P} / 2004$ A1 (LONEOS) (considering that the grain size is greater than the observing wavelength) and as probable for absorbing grains. A different value for $Q_{\mathrm{pr}}$ will determine a corresponding variation in the size. Of course, dust with size outside the range shown in Fig. 5 was also ejected from the inner coma, but smaller grains are outside the image in Fig. 4, while larger grains all fall in the nucleus pixel of Fig. 4. This panel of Fig. 5 confirms that all the observed tail must be composed of grains larger than $1 \mathrm{~cm}$ if the dust bulk density is $10^{3} \mathrm{~kg} \mathrm{~m}^{-3}$, or even larger than $10 \mathrm{~cm}$ in case of a more probable bulk density of $100 \mathrm{~kg} \mathrm{~m}^{-3}$. Assuming that all of the dust rate is due to CO sublimation, this upper size allows us to infer the lower value of the CO loss rate $Q$ that is necessary to lift up such a big grain. In case of uniform outgassing, the maximum size is (Crifo 1999)

$a_{\max }=1.57 \times 10^{-27} Q R_{\mathrm{n}}^{-3} \mathrm{~cm}$

where $R_{\mathrm{n}}$ is the radius of the nucleus (assumed to be a sphere) in km. We immediately get $Q>10^{30} \mathrm{~mol} \mathrm{~s}^{-1}$ if $R_{\mathrm{n}}=10 \mathrm{~km}$ and $a_{\max }>1.57 \mathrm{~cm}$. Such a CO loss rate is much larger than the steady one observed, as an example, in 29P/S-W 1 (Cochran et al. 1980; Senay \& Jewitt 1994; Festou et al. 2001). This high CO loss rate puts severe constraints on every model of Centaur nuclei.
Panel a of Fig. 5 shows the dust ejection velocity for the same dust size of $1 \mathrm{~cm}$ (assuming the standard dust bulk density of $10^{3} \mathrm{~kg} \mathrm{~m}^{-3}$ ). All the solutions with time-independent dust ejection velocity (values between 0.28 and $0.44 \mathrm{~m} \mathrm{~s}^{-1}$ ) derive from very good fits of the observed dust environment, as shown by the good fits in Fig. 4. We can conclude that a timeindependent dust ejection velocity is consistent with the observed neck-line.

The right-hand panels of Fig. 5 show the automatic outputs of the inverse dust tail model. In order to convert the 2D plot of the time and size-dependent distribution into a 1D one, for each time step we fitted the distribution versus size by a power law, the power index of which is plotted in panel b of Fig. 5, showing the time dependence of the dust size distribution. The output related to $u=-1 / 2$ is affected by strong noise, which obliged us to adopt larger regularizing weights to avoid output instabilities. Therefore, this solution is less probable than the others, when also taking into account that it is improbable that dust grains are perfect spheres. The outputs related to $u=-1 / 4$ and $u=-1 / 6$ show very good agreement with very small changes from the aphelion to the perihelion, in agreement with the expected steady conditions of a CO-driven dust environment. In particular, the power index of the dust size distribution is close to -3.5 for grains larger than $1 \mathrm{~cm}$, while it is close to -4.5 for grains smaller than $1 \mathrm{~cm}$ ejected at the perihelion. We recall that the dust tail contains data neither on grains smaller than $1 \mathrm{~cm}$ ejected around the aphelion nor on grains larger than $1 \mathrm{~cm}$ ejected around perihelion.

Panel d of Fig. 5 shows the $A f \rho$ value predicted by the model (Fulle 2005), which remains between 0.5 and $2 \mathrm{~m}$ from the aphelion to perihelion. It is quite close to the value measured on the TNG image (see Table 4): the inverse tail model computes the predicted perihelion $A f \rho$ value for dust sizes ranging from $0.2 \mathrm{~mm}$ to $2 \mathrm{~cm}$, so that it must be slightly lower than the observed value, which comes from all the grains with all possible dust sizes. The same panel shows the dust mass loss rate, which again remains almost constant between 100 and $200 \mathrm{~kg} \mathrm{~s}^{-1}$, an even higher value than was obtained by the same inverse dust tail model applied to Chiron (Fulle 1992). Therefore, all the outputs of the model confirm that a steady CO-driven dust environment is the most probable explanation of the observed dust tail and coma of P/2004 A1 (LONEOS). The real surprise is the extremely high level of both $\mathrm{CO}$ and dust loss rates, but such a result mainly depends on the fact that the dust tail must be composed of grains larger than $1 \mathrm{~cm}$, and this fact does not depend on debatable assumptions on dust parameters: it depends on the $\beta$ value provided by well-established orbit computations.

\section{Conclusions}

The outputs of the model described in previous section show that the Neck-line observed in TNG and CAHA images have been emitted many years (half an orbit) before the present time, i.e. very close to the Saturn encounter described in Sect. 4. We investigated whether the encounter could cause a strong tidal effect on the surface. The ratio between the gravitational acceleration on LONEOS nucleus, assumed to be a sphere, computed as

$g_{\mathrm{n}}=\frac{G \cdot M_{\mathrm{n}}}{R_{\mathrm{n}}^{2}}$

and the tidal acceleration due to the planet computed as

$a_{\tau}=\frac{2 \cdot G \cdot M_{\mathrm{P}} \cdot R_{\mathrm{n}}}{d^{3}}$ 
results in more than $3 \times 10^{5}$; here $G$ is the gravitational constant, $M_{\mathrm{n}}$ the nucleus mass, $R_{\mathrm{n}}$ the nucleus radius, $M_{\mathrm{P}}$ the mass of the planet (Saturn), and $d$ the minimum distance from Saturn. Note that the result only depends linearly on P/2004 A1 (LONEOS) density (here considered $10^{3} \mathrm{~kg} \mathrm{~m}^{3}$ ) and does not depend on its radius, and that even large uncertainties on this density cannot change this result; i.e. the close Saturn encounter cannot be considered responsible for any dust emission. As a comparison, during the close encounter of the comet Shoemaker-Levy 9 with Jupiter in 1995, the same ratio was 0.4, under the same assumptions.

A further check can be made by using the Roche Limit (RL), which is the distance at which a body with no tensile strength begins to be torn apart tidally by the nearby larger body. Approximately it is

$R L=R_{\mathrm{P}} \cdot\left(2 \cdot \frac{\rho_{\mathrm{P}}}{\rho_{\mathrm{n}}}\right)^{1 / 3}$

where $\rho_{\mathrm{P}}$ and $\rho_{\mathrm{n}}$ are the planet and nucleus densities, respectively, and $R_{\mathrm{P}}$ the planet radius. When applied to our cases, RL is 67 times smaller than the minimum P/2004 A1 (LONEOS) Saturn distance, while it is 1.4 times larger than the minimum distance for Shoemaker-Levy 9 - Jupiter.

A recent jump in the perihelion distance has been often linked to an onset of an increase in the dust and gas activity for short-period comets (Licandro et al. 2000). But to observe physical consequences of changes in dynamical parameters, at least one passage at the "new" perihelion distance should occur. This is not the case for $\mathrm{P} / 2004$ A1 (LONEOS), for which these are the first observations in the inner part of the Solar System. Therefore, the abundant dust production rate derived from the tail fit can be considered as the typical behaviour of the body also on the orbit before the Saturn encounter.

Direct radio measurements of CO contents in some Centaurs (Bockelée-Morvan et al. 2001) show an upper limit in the $\mathrm{CO}$ production rate of $10^{28} \mathrm{~mol} \mathrm{~s}^{-1}$. This value, put into Eq. (5), provides a radius of about $2 \mathrm{~km}$ for $\mathrm{P} / 2004 \mathrm{~A} 1$ (LONEOS). Actually, the radius of P/2004 A1 (LONEOS) has not been directly measured. Hahn et al. (2006) obtain an indirect measurement from prediscovery images of the Centaur taken in October 2003 from the Steward Observatory (Kitt Peak) (Marsden 2004). Assuming an average nuclear magnitude of $V=$ 20.7 and following the method applied in Tancredi et al. (2000), they derived a nucleus of $R_{\mathrm{n}}=9.2 \mathrm{~km}$. But this value can be considered only an upper limit of the nucleus radius, since the Centaur was not inactive at the time of prediscovery images: in fact, taking the PFOV of the prediscovery telescope into account and assuming a seeing for Kitt Peak observations of $0.5^{\prime \prime}$, Af $\rho=$ $490 \mathrm{~cm}$ is derived, that is even higher than the value derived for observations presented in this paper. A more relaxed seeing value of $0.8^{\prime \prime}$ would yield $A f \rho=250 \mathrm{~cm}$, which is compatible with values obtained by us when the Centaur was at closer heliocentric and geocentric distances.

Therefore, a nucleus as small as $2 \mathrm{~km}$ in radius could in principle be assumed for $\mathrm{P} / 2004$ A1(LONEOS). But could such a small nucleus sustain the observed activity for a long time? The dust production rate was estimated in $100 \mathrm{~kg} / \mathrm{s}$ (see Sect. 7), while a molecular production rate of $10^{28} \mathrm{~mol} \mathrm{~s}^{-1}$, assumed completely due to the $\mathrm{CO}$ molecule, turns in a volatile production rate of $\sim 470 \mathrm{~kg} / \mathrm{s}$, yielding a total production rate of $\sim 600 \mathrm{~kg} / \mathrm{s}$. With this mass loss rate, a $2 \mathrm{~km}$ radius body would be blown off in $\sim 2 \times 10^{3}$ years, orders of magnitude less than the commonly believed lifetimes of Centaurs. Therefore, if we assume a larger size, we infer the highest CO loss rate ever observed for the Centaur class.

Key measurements to solve the question would be:

(i) a direct estimation of the diameter of the body;

(ii) the direct observation of the $\mathrm{CO}$ production rate with radio measurements;

(iii) the direct IR spectroscopic measurements (with space telescopes) of the CO content in the Centaur coma.

Acknowledgements. Observations at the TNG were partially funded by the Fundación Galileo Galilei (INAF - Istituto Nazionale di Astrofisica). Observations at the $2.2 \mathrm{mt}$ of CAHA were funded by the Optical Infrared Coordination Network (OPTICON), a major international collaboration supported by the Research Infrastructures Programme of the European Commission's Sixth Framework Programme. We gratefully aknowledge funding from Italian Space Agency (ASI) under contract I/032/05/0.

\section{References}

A'Hearn, M. F., Schleicher, D. G., Feldman, P. D., Millis, R. L., \& Thompson, D. T. 1984, AJ, 89, 579

Barucci, M. A., de Bergh, C., Cuby, J.-G., et al. 2000, A\&A, 357, L53

Barucci, M. A., Boehnhardt, H., Dotto, E., et al. 2002, A\&A, 392, 335

Barucci, M. A., Doressoundiram, A., \& Cruikshank, D. P. 2005, in Comets II, ed. M. Festou, H. U. Keller, \& H. A. Weaver (Tucson: Univ. Arizona Press) Bauer, J. M., Meech, K. J., Fernandez, Y. R., Farnham, T. L., \& Roush, T. L. 2002, PASP, 114, 1309

Bauer, J. M., Fernandez, Y. R., \& Meech, K. J. 2003a, PASP, 115, 981

Bauer, J. M., Meech, K. J., Fernandez, Y. R., et al. 2003b, Icarus, 166, 195

Belskaya, I. N., Barucci, M. A., \& Shkuratov, Y. G. 2003, EM\&P, 92, 1, 201

Bernstein, G. M., Trilling, D. E., Allen, R. L., et al. 2004, AJ, 128, 1390

Bockelée-Morvan, D., Lellouch, E., Biver, N., et al. 2001, A\&A, 377, 343

Bockelée-Morvan, D., Crovisier, J., Mumma, M. J., \& Weaver, H. 2005, in Comets II, ed. M. Festou, H. U. Keller, \& H. A. Weaver (Tucson: Univ. Arizona Press)

Brown, M. E. 2000, AJ, 119, 977

Brown, M. E., \& Koresko, C. D. 1998, ApJ, 505, L65

Buzzi, L., Naves, R., Campas, M., et al. 2004, MPEC 2004-D23

Capria, M. T. 2002, EM\&P, 89, 161

Capria, M. T., Coradini, A., De Sanctis, M. C., \& Orosei, R. 2000a, A\&A, 357, 359

Capria, M. T., Coradini, A., De Sanctis, M. C., \& Orosei, R. 2000b, AJ, 119, 3112

Capria, M. T., Coradini, A., \& De Sanctis, M. C. 2002, EM\&P, 90, 1, 217

Cartwright, I. M., Fitzsimmons, A., \& Pollacco, D. L. 1999, EM\&P, 78, 1/3, 127

Choi, Y.-J., Prialnik, D., \& Brosch, N. 2002, Proc. IAU 8th AsianPacific Regional Meeting, ed. S. Ikecuhi, J. Hearnshaw, \& T. Hanawa (Astronomical Society of Japan)

Choi, Y.-J., Weissman, P. R., \& Polishook, D. 2006, IAU Circ., 8656

Cochran, A., Barker, E. S., \& Cochran, W. 1980, AJ, 85, 474

Coradini, A., Capaccioni, F., Capria, M. T., et al. 1997, Icarus, 129, 2, 317

Cremaschini, C., Foglia, S., Marinello, W., et al. 2004, MPEC 2004-E06

Crifo, J. F., Rodionov, A. V., \& Bockelée-Morvan, D. 1999, Icarus, 138, 1, 85

Crovisier, J., 1999, in Formation and Evolution of Solid in Space, ed. J. M. Greenberg, \& A. Li (Kluwer Academic Publisher), 39

Cruikshank, D. P., Roush, T. L., Bartholomew, M. J., et al. 1998, Icarus, 135, 389 Davies, J. K., Sykes, M. V., \& Cruikshank, D. P. 1993, Icarus, 102, 166

Delsemme, A. H. 1982, in Comets, ed. L. L. Wilkening (Univ. of AZ Press), 85 Descour, A. S., Skiff, B. A., Larson, S., et al. 2004, MPEC 2004-A51

Doressoundiram, A., Tozzi, G. P., Barucci, M. A., et al. 2003, AJ, 125, 2721

Doressoundiram, A., Barucci, M. A., Tozzi, G. P., et al. 2005, PSS 53, 14, 1501

Dotto, E., Barucci, M. A., \& De Bergh, C. 2003a, EM\&P 92, 1, 157

Dotto, E., Barucci, M. A., Boehnhardt, H., et al. 2003b, Icarus, 162, 2, 408

Dotto, E., Barucci, M. A., Leyrat, C., et al., 2003c, Icarus, 164, 1, 122

Duncan, M. J., \& Levison, H. F. 1997, Science, 276, 1670

Elliot, J. L., Kern, S. D., Clancy, K. B., et al. 2005, AJ, 129, 1117

Epifani, E., Colangeli, L., Fulle, M., et al. 2001, Icarus, 149, 339

Farnham, T. L., \& Schleicher, D. G. 2005, Icarus, 173, 533

Fernandez, Y. R., Meech, K. J., Pittichova, J., et al. 2001, IAU Circ., 7689

Festou, M. C., Gunnarsson, M., Rickman, H., Winnberg, A., \& Tancredi, G. 2001, Icarus, 150,140

Fornasier, S., Doressoundiram, A., Tozzi, G. P., et al. 2004, A\&A, 421, 353

Fulle, M. 1989, A\&A, 217, 283

Fulle, M. 1992, Nature, 359, 6390, 42

Fulle, M. 1994, A\&A, 282, 980 
Fulle, M. 2005, in Comets II, ed. M. Festou, H. U. Keller, \& H. A. Weaver (Tucson: Univ. Arizona Press)

Fulle, M., Bosio, S., Cremonese, G., et al. 1993, A\&A, 272, 634

Fulle, M., Barbieri, C., Cremonese, G., et al. 2004, A\&A, 422, 357

Green, D. W. E. 2004, IAU Circ., 8430

Green, D. W. E. 2005, IAU Circ., 8618

Hahn, G., Lagerkvist, C.-I., Karlsson, O., Oja, T., \& Stoss, M. 2006, Astron. Nachr., 327, 1, 17

Hainaut, O. R., Delahodde, C. E., Boehnhardt, H., et al. 2000, A\&A, 356, 1076

Hartmann, W. K., Tholen, D. J., Meech, K. J., \& Cruikshank, D. P. 1990, Icarus, 83,1

Holman, M. J., \& Wisdom, J. 1993, AJ, 105, 5, 1987

Jenniskens, P., Blake, D. F., \& Kouchi, A. 1998, in Solar System Ices, Proceedings of the Symposium held in Toulouse, March 27-30, 1995, ASSL, 227 (Dordrecht: Kluwer Academic Publ.)

Jewitt, D. C. 1990, ApJ, 351, 277

Kimura, H., \& Liu, C. P. 1977, Chin. Astron., 1, 235

Krishna Swamy, K. S. 1986, Physics of comets (World Scientific Pub.)

Kronk, G. 2005, Electronic catalogue of periodic comets, http://cometography.com/periodic_comets.htm

Kusnirak, P., \& Balam, D. 2000, IAU Circ., 7368

Landolt, A. U. 1992, AJ, 104, 1, 340

Lara, L.-M., Licandro, J., Oscoz, A., \& Motta, V. 2003, A\&A, 399, 763

Lara, L.-M., Tozzi, G. P., Boehnhardt, H., DiMartino, M., \& Schulz, R. 2004, A\&A, 422, 717

Larsen, J. A., Gleason, A. E., Danzi, N. M., et al. 2001, AJ, 121, 562

Laufer, D., Kochavi, E., \& Bar-Nun, A. 1987, Phys. Rev. B, 36, 9219

Lazzarin, M., Barucci, M. A., Boehnhardt, H., et al. 2003, AJ, 125, 1554

Lawrence, K. J., Helin, E. F., Pravdo, S., Dyvig, R., \& Balam, D. D. 2001, IAUC Circ., 7654

Levison, H. F., \& Duncan, M. J. 1997, Icarus, 127, 13

Licandro, J., Tancredi, G., Lindgren, M., Rickman, H., \& Gil Hutton, R. 2000, Icarus, 147,161

Lowry, S. C., \& Fitzsimmons, A. 2001, A\&A, 365, 204

Lowry, S. C., \& Fitzsimmons, A. 2005, MNRAS, 358, 641

Lowry, S. C., \& Weissmann, P. R. 2003, Icarus, 164, 492

Lowry, S. C., Fitzsimmons, A., Cartwright, I. M., \& Williams, I. P. 1999, A\&A, 349, 649

Lowry, S. C., Fitzsimmons, A., \& Collander-Brown, S. 2003, A\&A, 397, 329

Luu, J. X., \& Jewitt, D. C. 1990, AJ, 100, 913

Luu, J. X., Jewitt, D. C., \& Cloutis, E. 1994, Icarus, 109, 133
Luu, J. X., Jewitt, D. C., \& Trujillo, C. 2000, ApJ, 531, L151

Marsden, B. 1962, PASP, 8, 375

Marsden, B. 2004, MPEC 2004-B27

Meech, K., \& Belton, M. 1989, IAU Circ., 4770

Meech, K. J., \& Svoren, J. 2005, in Comets II, ed. M. Festou, H. U. Keller, \& H. A. Weaver (Tucson: Univ. Arizona Press)

Meech, K. J., Hainaut, O. R., \& Marsden, B. G. 2004, Icarus, 170, 463

Merlin, F., Barucci, M. A., Dotto, E., de Bergh, C., \& Lo Curto, G. 2005, A\&A, 444, 977

Millis, R. L., Buie, M. W., Wasserman, L. H., et al. 2002, AJ, 123, 2083

Naves, R., Campas, M., Kadota, K., et al. 2004, MPEC 2004-C07

Peixinho, N., Boehnhardt, H., Belskaya, I., et al. 2004, Icarus, 170, 153

Petit, J.-M., \& Gladman, B. 2003, C.R. Phys., 4, 743

Petit, J.-M., Holman, M. J., Gladman, B., et al. 2006, MNRAS, 365, 429

Pravdo, S., Helin, E. F., Hicks, M., \& Lawrence, K. 2001, IAU Circ., 7738

Prialnik, D., Brosch, N., \& Ianovici, D. 1995, MNRAS, 276, 4, 1148

Read, M. T., \& Scotti, J. V. 2005, IAU Circ., 8614

Rousselot, P., Petit, J.-M., Poulet, F., \& Sergeev, A. 2005, Icarus, 176, 478

Schleicher, D. G., Woodney, L. M., \& Millis, R. L. 2003, Icarus, 162, 2, 415

Schulz, R., Stüwe, J. A., Boehnhardt, H., Gaessler, W., \& Tozzi, G. P. 2003, A\&A, 398, 345

Senay, M. C., \& Jewitt, D. C. 1994, Nature, 371, 229

Sheppard, S. S., Jewitt, D. C., Trujillo, C. A., Brown, M. J. I., \& Ashley, M. C. B. 2000, AJ, 120, 2687

Skiff, B. A., Miller, H. R., \& Young, J. 2004, IAU Circ., 8267

Szabó, G. M., Csák, B., Sárneczky, K., \& Kiss, L. L. 2001, A\&A, 374, 712

Szabó, G. M., Kiss, L. L., Sárneczky, K., \& Sziládi, K. 2002, A\&A, 384, 702

Tancredi, G., Fernandez, J. A., Rickman, A., \& Licandro, J. 2000, A\&AS, 146, 73

Tegler, S. C., Romanishin, W., Descour, A. S., \& Marsden, B. G. 2005, MPEC 2005-M12

Tesi, L., Boattini, A., Tombelli, M., et al. 2004, MPEC 2004-C46

Ticha, J., Tichy, M., Kadota, K., et al. 2004, MPEC 2004-B54

Trujillo, C. A., Luu, J. X., Bosh, A. S., \& Elliot, J. L. 2001, AJ, 122, 2740

UAI 2006, http://comete.uai.it/

Weiler, M., Rauer, H., Knollemberg, J., Jorda, L., \& Helbert, J. 2003, A\&A, 403, 313

Weiler, M., Rauer, H., \& Helbert, J. 2004, A\&A, 414, 749

Womack, M., \& Stern, A. S. 1999, Astron. Vestnik, 33, 187

Yu, Q., \& Tremain, S. 1999, AJ, 118, 1873 